\title{
The ejecta of $\eta$ Carinae
}

\section{Theodore R. Gull}

Exploration of the Universe Division, Laboratory for Extrasolar Planets and Stellar Astrophysics, Code 667, Goddard Space Flight Center, Greenbelt, MD 20771, USA email: theodore.r.gull@nasa.gov

\begin{abstract}
High-dispersion spectroscopic observations of the neutral Homunculus and the ionized Little Homunculus, ejecta of $\eta$ Car, are being analyzed to determine the relative abundances of metals. Thousands of lines of neutral and singly-ionized metals and molecules seen in the Homunculus suggest that this oxygen-, carbon-poor, nitrogen-, helium-rich gas contains very different dust grains likely devoid of metal oxides. The gas to dust ratio is likely much larger than the canonical 100:1 implying that the $12 \mathrm{M}_{\odot}$ estimate of the ejecta is a lower limit.
\end{abstract}

Keywords. stars: individual ( $\eta$ Car), winds, mass loss, abundances, binaries

High-dispersion spectra of $\eta$ Car recorded with the HST-STIS and the VLT-UVES are filled with thousands of narrow absorption lines with several different velocity systems (Gull et al. 2006). Two well-isolated, dominant velocity systems correspond to the Homunculus $\left(-513 \mathrm{~km} \mathrm{~s}^{-1}, 760 \mathrm{~K}, 10^{6-7} \mathrm{~cm}^{-3}\right)$, ejected in the $1840 \mathrm{~s}$ and the Little Homunculus $\left(-146 \mathrm{~km} \mathrm{~s}^{-1}, 6400 \mathrm{~K}, 10^{7} \mathrm{~cm}^{-3}\right.$ ) associated with the 1890s lesser event (Gull et al. 2005). In the $-513 \mathrm{~km} \mathrm{~s}^{-1}$ gas, lines of Fe I, Fe II, Ni II II, Cr II, Ti II, V II, Sr II, Sc II, Mn II, Mg II, NaI, etc., (with ionization potentials less than $8 \mathrm{eV}$ ), are found along with nearly a thousand lines of $\mathrm{H}_{2}$, plus several lines of $\mathrm{CH}, \mathrm{OH}, \mathrm{NH}$, and $\mathrm{CH}+\left(-513 \mathrm{~km} \mathrm{~s}^{-1}\right.$, but $60 \mathrm{~K}, 10^{7} \mathrm{~cm}^{-3}$ ), but no $\mathrm{CO}$. The $\mathrm{H}_{2}$ absorption lines originate from very high rotational levels and are characteristic of UV photo-excitation/dissociation, are present during $\eta$ Car's periodic broad (5 year) maximum but disappear during the few month long low-excitation minimum. This suggests a layered structure with transition from partially ionized gas to a neutral gas with molecular and dust formation. IR flux measures (Smith et al. 2003) imply a total ejection mass of $\sim 12 \mathrm{M}_{\odot}$.

Initial measures of ionic and neutral column densities of this gas and the Strontium Filament, a partially ionized emission nebula in the skirt of the Homunculus, demonstrate that gaseous $\mathrm{Ti} / \mathrm{Ni}, \mathrm{Cr} / \mathrm{NI}, \mathrm{V} / \mathrm{Fe}$ are much more than solar, indicating that most metals remain suspended in be of silicates and alumina (Chesneau et al. 2005). Additional abundance ratios, but are constrained by the need for improved laboratory measurements and/or theoretical modeling of $g f$-values for these species.

\section{Acknowledgements}

Observations for this project were accomplished with the NASA/ESA Hubble Space Telescope-STIS and the ESO Very Large Telescope-UVES.

\section{References}

Chesneau, O., Verhoelst, T., Lopez, B., et al. 2005, A\&AA, 435, 563

Gull, T. R., Vieira Kober, G., Bruhweiler, F., et al. 2005, ApJ, 620, 442

Gull, T. R., Vieira Kober, G., \& Nielsen, K. E. 2006, ApJS, 163, 173

Smith, N., Gehrz, R. D., Hinz, P. M., et al. 2003, AJ, 125, 1458 